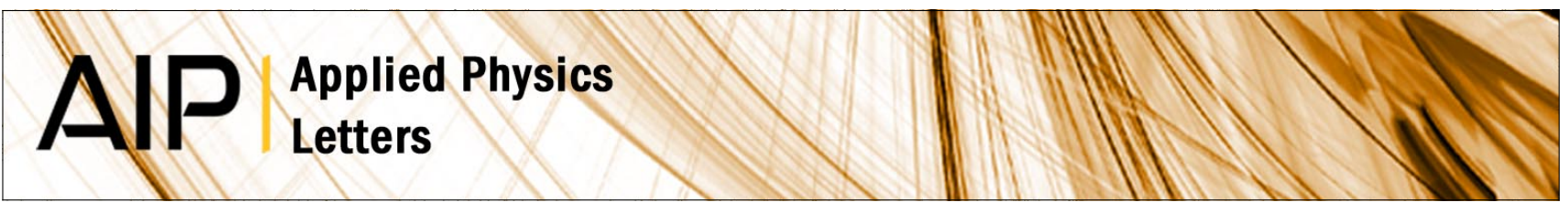

\title{
Nonlinear dynamics of relativistic charged particle beams
}

R. P. Nunes and F. B. Rizzato

Citation: Appl. Phys. Lett. 98, 051501 (2011); doi: 10.1063/1.3549690

View online: http://dx.doi.org/10.1063/1.3549690

View Table of Contents: http://apl.aip.org/resource/1/APPLAB/v98/i5

Published by the American Institute of Physics.

\section{Related Articles}

Meissner effect funneling

Appl. Phys. Lett. 99, 054101 (2011)

Observation of coherently enhanced tunable narrow-band terahertz transition radiation from a relativistic subpicosecond electron bunch train

Appl. Phys. Lett. 98, 261501 (2011)

Characterization and focusing of light ion beams generated by ultra-intensely irradiated thin foils at the kilojoule scale

Phys. Plasmas 18, 056713 (2011)

Novel Hamiltonian method for collective dynamics analysis of an intense charged particle beam propagating through a periodic focusing quadrupole lattice

Phys. Plasmas 18, 056712 (2011)

Generalized Courant-Snyder theory and Kapchinskij-Vladimirskij distribution for high-intensity beams in a coupled transverse focusing lattice

Phys. Plasmas 18, 056708 (2011)

\section{Additional information on Appl. Phys. Lett.}

Journal Homepage: http://apl.aip.org/

Journal Information: http://apl.aip.org/about/about_the_journal

Top downloads: http://apl.aip.org/features/most_downloaded

Information for Authors: http://apl.aip.org/authors

\section{ADVERTISEMENT}

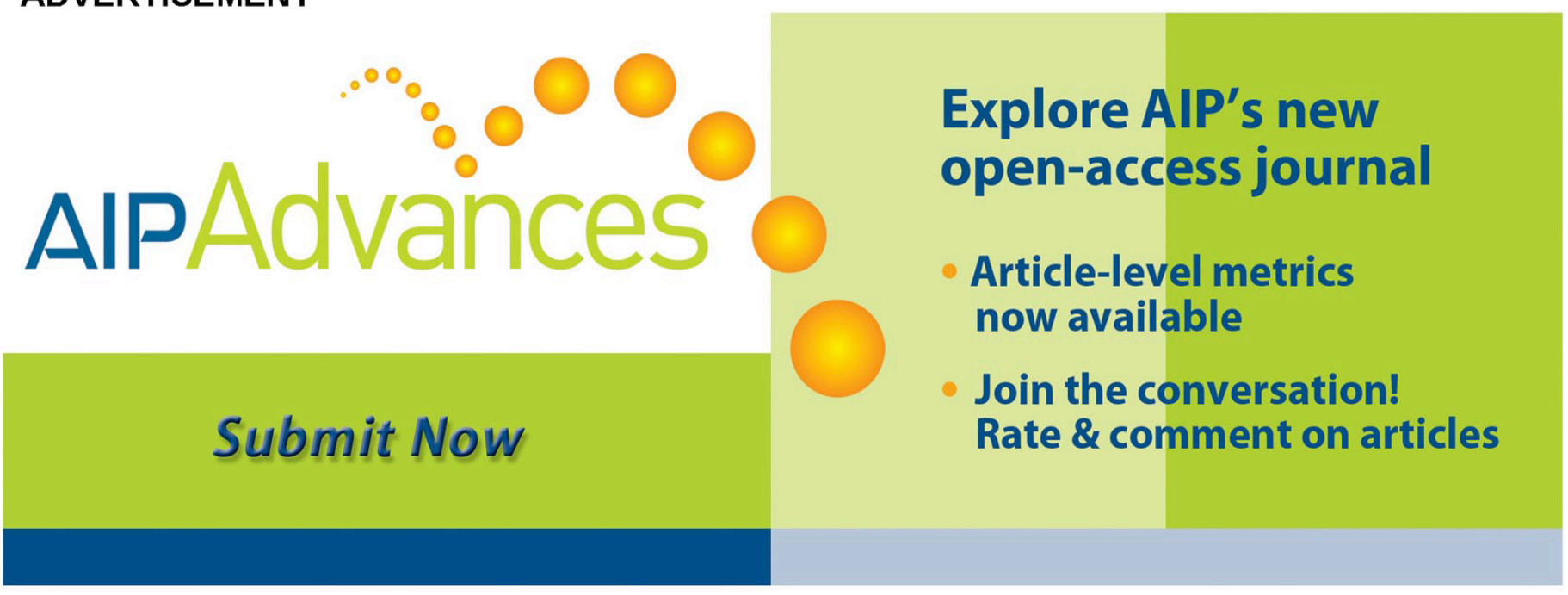




\title{
Nonlinear dynamics of relativistic charged particle beams
}

\author{
R. P. Nunes ${ }^{1, a)}$ and F. B. Rizzato ${ }^{2}$ \\ ${ }^{1}$ Instituto de Física e Matemática, Universidade Federal de Pelotas, P.O. Box 354, Pelotas, \\ Rio Grande do Sul 96010-900, Brazil \\ ${ }^{2}$ Instituto de Física, Universidade Federal do Rio Grande do Sul, P.O. Box 15051, Porto Alegre, \\ Rio Grande do Sul 91501-970, Brazil
}

(Received 9 June 2010; accepted 5 January 2011; published online 31 January 2011)

\begin{abstract}
The idea behind this work is to analyze the transversal dynamics of a relativistic charged particle beam. The beam is azimuthally symmetric, focused by a constant magnetic field and supposed to be initially cold. While mismatched, nonrelativistic, and homogeneous beams oscillate with an invariant cold density profile, it is shown that relativistic homogeneous beams progressively heat and lose an important amount of constituents during its magnetic confinement. This heating process starts with phase-space wave-breaking, a mechanism observed before in initially inhomogeneous beams. The results have been obtained with full self-consistent $N$-particle beam numerical simulations. (C) 2011 American Institute of Physics. [doi:10.1063/1.3549690]
\end{abstract}

Beams composed of charged particles usually evolve to equilibrium as they eject a representative amount of its constituents in the focusing channel. The ejected particles form a rarified population around a denser one in the beam phasespace. While the first population is recognized as the beam halo, the second one is denoted as the beam core. ${ }^{1-4} \mathrm{~A}$ halo has many undesired implications on the accelerator structure, reducing beam lifetime and increasing maintenance costs. ${ }^{5}$ Also, there are many applications in which beam halo has to be, if not suppressed, at least minimized. ${ }^{6}$

The way beam particles self-consistently interact and generate a halo depends on the beam initial distribution. ${ }^{7}$ For initially cold, quasihomogeneous, and mismatched beams, it has been found that, while the initial spurious inhomogeneity is the forerunner mechanism that allows particles to be progressively excited, in fact the envelope mismatch is the mechanism effectively responsible for halo formation. ${ }^{8}$ On the other hand, for initially cold nonhomogeneous but envelope matched beams, it has been found that the forerunner mechanism by which particles are ejected from the core is through phase-space wave-breaking. ${ }^{9}$ Once out of the beam core, the ejected particles are permanently excited by a process called charge redistribution. ${ }^{8}$ If in this last situation the mismatch is also present, then envelope oscillation acts as another source of energy to excite beam particles. ${ }^{10}$ The only difference is that now the coupling is resonant. ${ }^{8}$

In the cases mentioned above, nothing has been commented about the relativity of the beam dynamics. In fact, a paraxial approximation has been considered, which implies that, although the beam could be relativistic in the longitudinal direction, its transversal dynamics was purely classic and nonrelativistic. However, notwithstanding the many situations in which this is an adequate approximation, in many others one can assure that this is not or have to be reformulated to include the desired relativistic effects. The mass correction introduced by the relativistic effects can be more or less impacting on the dynamics of beam particles and must be understood. For this purpose, hereafter, an analytical de-

${ }^{a)}$ Electronic mail: roger.pizzato@ufrgs.br. scription for the relativistic dynamics of beam particles will be presented.

Suppose an initially homogeneous beam of charged particles evolving inside a linear channel. A conducting pipe with a circular transversal section encapsulates the channel. Solenoids provide the constant magnetic field that permeates the channel. The beam has a constant velocity $\dot{z}$ along the pipe symmetry axis, which can be also recognized as axis $z$. For this system, from Newton's second law, the dynamics of beam particles could be described by the following equation $^{11}$

$$
\frac{d}{d t} \mathbf{P}=\mathbf{F}_{\text {lorentz }}=q(\mathbf{E}+\mathbf{v} \times \mathbf{B}),
$$

in which $\mathbf{P}=\gamma m \mathbf{v}$ is the relativistic linear momentum, $\mathbf{v}$ is the velocity of the particle, $\gamma$ is the Lorentz factor, $q$ is the charge and $m$ is the rest mass of the particle, and $\mathbf{E}$ and $\mathbf{B}$ are, respectively, the electric and magnetic flux density fields inside the accelerator structure.

Since the beam here is azimuthally symmetric, Eq. (1) could be expressed with cylindrical coordinates. Consider $\left(\mathbf{e}_{r}, \mathbf{e}_{\theta}, \mathbf{e}_{z}\right)$ as the unit-vector base in cylindrical coordinates. In this case, $\mathbf{v}=\dot{r} \mathbf{e}_{r}+r \dot{\theta} \mathbf{e}_{\theta}+\dot{z} \mathbf{e}_{z}$ and the fields $\mathbf{E}=E_{r} \mathbf{e}_{r}+E_{\theta} \mathbf{e}_{\theta}$ $+E_{z} \mathbf{e}_{z}$ and $\mathbf{B}=B_{r} \mathbf{e}_{r}+B_{\theta} \mathbf{e}_{\theta}+B_{z} \mathbf{e}_{z}$. Then, in this coordinate system, Eq. (1) has the following form:

$$
\begin{aligned}
& \frac{d}{d t}(\gamma m \dot{r})-\gamma m r \dot{\theta}^{2}=q\left(E_{r}+r \dot{\theta} B_{0}-\dot{z} B_{\theta}\right), \\
& \frac{1}{r} \frac{d}{d t}\left(\gamma m r^{2} \dot{\theta}\right)=-q \dot{r} B_{0}, \\
& \frac{d}{d t}(\gamma m \dot{z})=q \dot{r} B_{\theta},
\end{aligned}
$$

in which, due to the symmetry of the problem, it has that $E_{\theta}=E_{z}=B_{r}=0$, and $B_{z}=B_{0} . B_{0}$ is the density of magnetic flux applied by the solenoids. $E_{r}$ and $B_{\theta}$ are the fields generated self-consistently by the particle distribution assigned to the beam. The notation $\dot{x}$ means $d x / d t$ with $x=\{r, \theta, z\}$. In the limit $\gamma \rightarrow 1$, the system of ordinary differential equation 
(ODE) in Eq. (2) reduces to that of a nonrelativistic case. Solving the ODE for the $\mathbf{e}_{\theta}$ component, one obtains

$$
\gamma m \dot{\theta}=-\frac{q B_{0}}{2}=\text { const. }
$$

Note that $\gamma m \dot{\theta}$ is now a conserved quantity. The Larmor frequency $\dot{\theta}$ of particles in the external density of magnetic field is a function of the absolute value of velocity $\mathbf{v}$. With the aid of Eq. (3) and supposing a changing of variables of $t$ to $z$, one can rewrite the ODE for the $\mathbf{e}_{r}$ component of Eq. (1) as follows:

$$
\frac{d^{2} r}{d z^{2}}+\frac{\dot{\theta}^{2}}{\dot{z}^{2}} r=\frac{q}{\gamma m \dot{z}^{2}}\left[E_{r}-\dot{z} B_{\theta}-\dot{z} B_{\theta}\left(\frac{d r}{d z}\right)^{2}\right],
$$

in which also has been used the ODE for the $\mathbf{e}_{z}$ component of Eq. (1).

To turn Eq. (4) completely solvable, it is necessary to determine both $E_{r}$ and $B_{\theta}$. Considering that initially all charged particles have negligible velocity, and with $n_{b}$ being the initial beam density, from the Ampere-Maxwell law, $\nabla$ $\times \mathbf{B}=\mu_{0} \mathbf{J}+\left(1 / c^{2}\right) \partial \mathbf{E} / \partial t$, bearing in mind the symmetry of the problem and the stationary characteristic of the fields, the previous equation reduces to

$$
B_{\theta}=\frac{\mu_{0} q \dot{z}}{2 \pi r} Q(r),
$$

in which $\mu_{0}$ is the vacuum magnetic permeability. $Q(r)$ $=\int_{0}^{r} n_{b}\left(r^{*}\right) r^{*} d r^{*} d \theta$ is the charge trapped by a Gauss surface at $r$. For the $E_{r}$ field, solving the Gauss law $\nabla \cdot \mathbf{E}=\rho / \epsilon_{0}$, one obtains

$$
E_{r}=\frac{q}{2 \pi r \epsilon_{0}} Q(r)
$$

in which $\rho$ is the beam density of charge and $\epsilon_{0}$ is the electric permittivity of the vacuum.

Observe that in fact $B_{\theta}=B_{\theta}(Q)$ and $E_{r}=E_{r}(Q)$, being possible to express $B_{\theta}$ as a function of $E_{r}$. Proceeding in this way,

$$
B_{\theta}=\frac{\dot{z}}{c^{2}} E_{r}
$$

Proposing the following change of variables

$$
\bar{z}=\gamma_{z} z, \quad \bar{r}=\beta_{z} r,
$$

and inserting the expression found in Eq. (7) into Eq. (4) with the help provided by Eq. (3), in view of that $\gamma=\gamma(z)$ by definition, after some extensive algebra, it is possible to achieve the equation

$$
\frac{d^{2} \bar{r}}{d \bar{z}^{2}}+\kappa_{0} \frac{1-\left(\frac{d \bar{r}}{d \bar{z}}\right)^{2}}{1+\kappa_{0} \bar{r}^{2}} \bar{r}=K \frac{\left[1-\left(\frac{d \bar{r}}{d \bar{z}}\right)^{2}\right]^{3 / 2}}{\left(1+\kappa_{0} \bar{r}^{2}\right)^{1 / 2}} \frac{Q(\bar{r})}{\bar{r}},
$$

in which

$$
\kappa_{0}=\frac{q^{2} B_{0}^{2}}{4 m^{2} \dot{z}^{2}}
$$

is the coefficient of magnetic focusing established by the solenoids, and
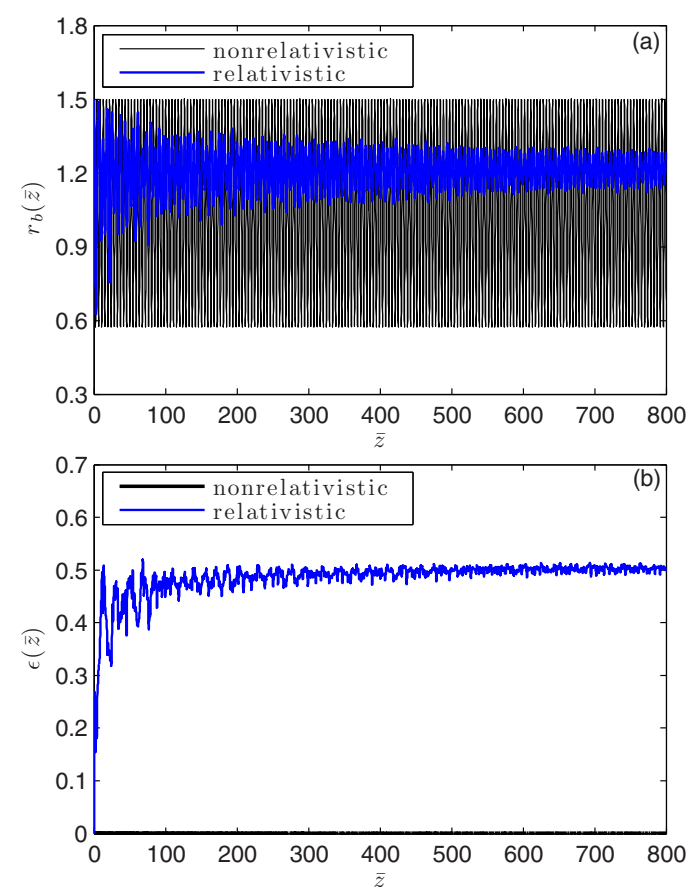

FIG. 1. (Color online) The results of numerical simulations for an initially $50 \%$ mismatched, cold, and homogeneous beam. (a) The beam envelope $r_{b}$ and (b) the beam emittance $\epsilon$. While nonrelativistic beams oscillate with fixed amplitude, relativistic beams suffer a strong decay. The emittance growth associated with the relativistic case is pretty perceptible. The beam is composed of $N_{b}=10000$.

$$
K_{0}=\frac{q^{2}}{2 \pi \epsilon_{0} m \gamma_{z} \dot{z}^{2}}
$$

is conventionally denominated the perveance of the beam, with $\gamma_{z} \equiv 1 / \sqrt{1-\beta_{z}^{2}}$ and $\beta_{z}=\dot{z} / c$. Once $\dot{z}=$ const, from the point of view of equations of motion, the axial length and time have the same meaning. Note that Eq. (9) is a completely nonlinear equation for the transversal dynamics of beam particles. The second order derivative depends not only on nonlinear functions of $\bar{r}$ but also on $d \bar{r} / d \bar{z}$. The term left to the right of Eq. (9) can be identified as the source for the particle transversal dynamics since the fields autoconsistently generated by the evolution of density $n_{b}$ excite each beam constituent.

To explore this information introduced by relativity, a full self-consistent $N$-particle beam numerical simulation is performed, with each particle being governed by Eq. (9). As the initial condition, the beam particle density is considered completely homogenous $n_{b}(\bar{r}, \bar{z}=0)=N_{b} / \pi r_{b}^{2}$, in which $r_{b}$ is the beam border, the envelope. For each particle $i$ at the radial coordinate $\bar{r}=\bar{r}_{i}, Q\left(\bar{r}_{i}\right)$ is self-consistently computed. Indeed, $Q\left(\bar{r}_{i}\right)$ represents the collective influences of all beam particles on the one at $\bar{r}_{i}$. The total number of beam particles adopted was $N_{b}=10000$.

Figure 1 shows the results obtained with the numerical simulations for two macroscopic beam quantities of interest: beam envelope in Fig. 1(a) and beam emittance, defined as $\epsilon=\sqrt{4\left(\left\langle\bar{r}^{\prime 2}\right\rangle\left\langle\bar{r}^{2}\right\rangle-\left\langle\bar{r}^{\prime} \vec{r}\right\rangle\right)}$ with $\bar{r}^{\prime}=d \bar{r} / d \bar{z}$, in Fig. 1(b). The angular brackets \langle\rangle represent phase-space averaging. The beam has been supposed to be initially mismatched by $50 \%, r_{0}$ $\equiv r_{b}(\bar{z}=0)=1.5$, since $r_{\mathrm{eq}}=1$. As expected, beam envelope for the nonrelativistic case oscillates over long axial lengths of the focusing channel with invariant amplitude. On the other 

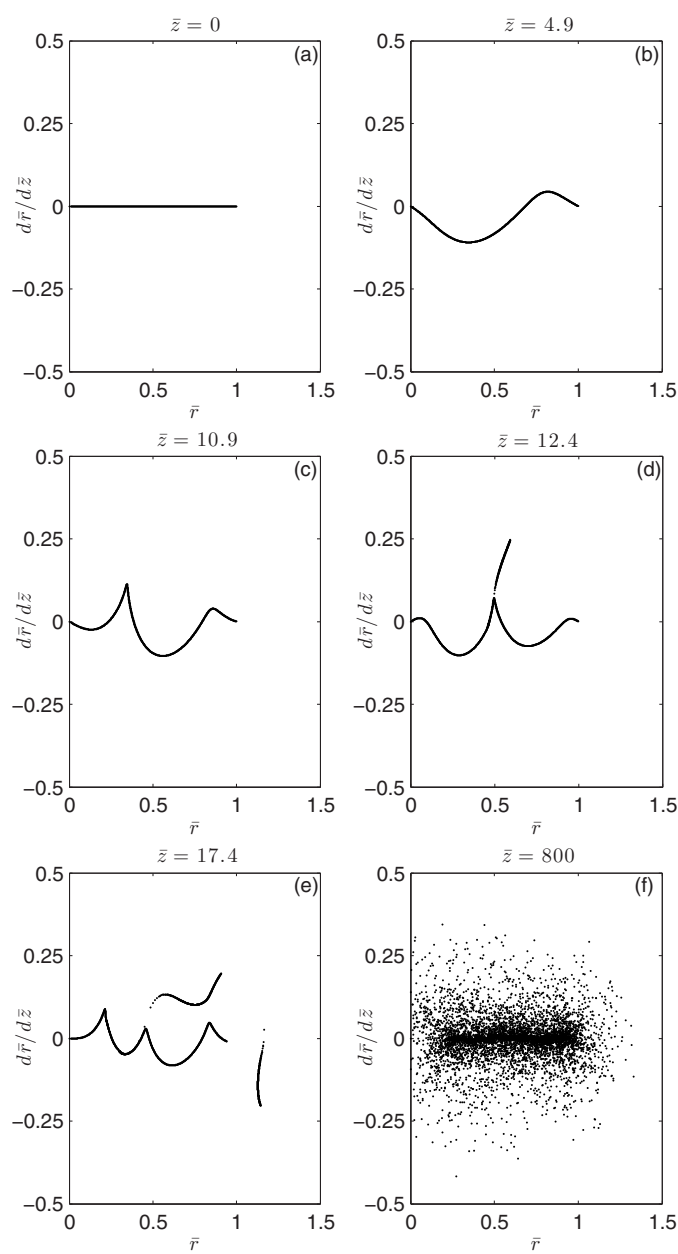

FIG. 2. A sequence of snapshots of the beam phase-space dynamics of an initially cold and relativistic beam with matched envelope. (a) The initial beam picture at the phase-space. During its evolution inside the focusing channel, beam charge starts to be redistributed as (b) shows. This process is amplified, and the phase-space density wave across the beam breaks. (c) shows the axial coordinate just before and (d) just after the first breaking. Many other wave-breakings occur during the beam confinement process [(e) shows the second] until reaching its equilibrium in (f).

side, the same does not occur if, for this same beam, the relativistic effects are considered. The beam envelope oscillates over shorter axial distance $\bar{z}$ until it suffers a sharp decay. In Fig. 1(b), it is possible to observe that, at this same coordinate, beam emittance experiences a sudden growth. The emittance growth is a macroscopic indicator that microscopic particles are increasing their velocities and enlarging the amplitude of their orbits in the phase-space. The particles with these attributes are potentially the ones that compose the beam halo and must be investigated in detail.

For this purpose, the beam phase-space dynamics is shown in Fig. 2, which has been composed of a set of snapshots at adequate axial lengths $\bar{z}$. In this case, the beam has been considered initially matched for practical purposes. The beam is initially cold, implying that at $\bar{z}=0$ its appearance at the phase-space is a horizontal line over the $\bar{r}$ axis [Fig. 2(a)]. However, as the beam propagates inside the focusing channel, a process of redistribution of charges is observed as Fig. 2(b) shows. Although initially particles are disposed homogeneously, during its dynamics, particles can accumulate differently along the beam. This gives rise to the propagation of waves inside the beam density. These phase-space waves are self-consistently amplified until a breaking is observed. Some particles are violently ejected from the beam core. Figures 2(c) and 2(d), respectively, show the axial length right before and right after the first breaking. Figure 2(e) shows the beam phase-space picture after the second breaking. Many other wave-breakings occur until the beam reaches its equilibrium, which is presented in Fig. 2(f).

This is interesting since wave-breaking has been observed before in just initially inhomogeneous beams. However, it is shown that also even initially homogeneous beams can undergo wave-breaking if the relativistic effects are not neglected. And this is of great importance for engineering purposes once particles expelled with the wave-breaking will be the ones that will form the beam halo.

In fact, as a general finding, wave-breaking is associated with the nonlinear behavior of the dynamical equation that describes the motion of each beam particle. The nonlinearity can be introduced by inhomogeneity or by including the relativistic effects to the description of the beam dynamics. Recent results have shown that for some particular kind of initial beam density, the inhomogeneity can compensate the relativistic effects and the wave-breaking phenomenon can be suppressed. ${ }^{12}$ But this is out of the scope of the present work and will be a subject of future works.

The authors acknowledge the aid of the National Centre of Supercomputation of the Brazil's South Region (CESUP), located at the Federal University of Rio Grande do Sul (UFRGS). This work has received financial support from the Conselho Nacional de Desenvolvimento Científico e Tecnológico $(\mathrm{CNPq})$, from the Fundação de Amparo à Pesquisa do Estado do Rio Grande do Sul (FAPERGS), both from Brazil, and from U.S.-AFOSR under Grant No. FA9550-091-0283.

${ }^{1}$ A. Cuchetti, M. Reiser, and T. Wangler, in Proceedings of the Invited Papers, 14th Particle Accelerator Conference, San Francisco, California, 1991, edited by L. Lizama and J. Chew (IEEE, New York, 1991), Vol. 1, p. 251; M. Reiser, J. Appl. Phys. 70, 1919 (1991).

${ }^{2}$ J. S. OConnell, T. P. Wangler, R. S. Mills, and K. R. Crandall, Proceedings of the Particle Accelerator Conference, Washington, D.C. (IEEE, New York, 1993), p. 3657.

${ }^{3}$ R. A. Jameson, Proceedings of the Particle Accelerator Conference, Washington, D.C. (IEEE, New York, 1993), p. 3926; Los Alamos National Laboratory Report No. LA-UR-93-1209, 1993.

${ }^{4}$ R. P. Nunes, R. Pakter, and F. B. Rizzato, Phys. Plasmas 14, 023104 (2007); J. Appl. Phys. 104, 013302 (2008).

${ }^{5}$ J. M. Lagniel, Nucl. Instrum. Methods Phys. Res. A 345, 46 (1994); 345, 405 (1994).

${ }^{6}$ T. Wangler, RF Linear Accelerators (Wiley-VCH, Darmstadt, 2008).

${ }^{7}$ C. K. Allen, K. C. D. Chan, P. L. Colestock, K. R. Crandall, R. W. Garnett, J. D. Gilpatrick, W. Lysenko, J. Qiang, J. D. Schneider, M. E. Schulze, R. L. Sheffield, H. V. Smith, and T. P. Wangler, Phys. Rev. Lett. 89, 214802 (2002).

${ }^{8}$ R. P. Nunes and F. B. Rizzato, Proceedings of the 23rd Particle Accelerator Conference, Vancouver, British Columbia (IEEE, Vancouver, 2009).

${ }^{9}$ F. B. Rizzato, R. Pakter, and Y. Levin, Phys. Plasmas 14, 110701 (2007); E. G. Souza, A. Endler, R. Pakter, F. B. Rizzato, and R. P. Nunes, Appl. Phys. Lett. 96, 141503 (2010).

${ }^{10}$ R. L. Gluckstern, Phys. Rev. Lett. 73, 1247 (1994).

${ }^{11} \mathrm{M}$. Reiser, Theory and Design of Charged Particle Beams (Wiley-VCH, Weinheim, 2004).

${ }^{12}$ R. P. Nunes and F. B. Rizzato, Proceedings of the 15th International Congress on Plasma Physics, Santiago, 8 August 2010. 